\title{
COMPLEX REPRESENTATION THEORY OF THE ELECTROMAGNETIC FIELD
}

\author{
ANDREAS ASTE
}

Communicated by Abraham Ungar

Abstract. A concise discussion of the three-dimensional irreducible $(1,0)$ and $(0,1)$ representations of the restricted Lorentz group and their application to the description of the electromagnetic field is given. It is shown that a mass term is in conflict with relativistic invariance of a formalism using electric and magnetic fields only, contrasting the case of the two-component Majorana field equations. An important difference between the Dirac equation and the Dirac form of Maxwell's equations is highlighted by considering the coupling of the electromagnetic field to the electric current.

\section{Introduction}

Starting from Lorentz symmetry as the key property of Minkowski space-time in the framework of the special theory of relativity, we may observe that the classical electric and magnetic field can be combined into a single photon wave function [3]

$$
\boldsymbol{\Psi}=\frac{1}{\sqrt{2}}(\mathbf{E}+\mathrm{i} \mathbf{B}), \quad \mathrm{i}^{2}=-1
$$

where the electric field $\mathbf{E}$ and the magnetic field $\mathbf{B}$ are three-component real fields which, for the sake of convenience, shall be written in column matrix form

$$
\mathbf{E}(x)=\left(\begin{array}{c}
E_{1}(x) \\
E_{2}(x) \\
E_{3}(x)
\end{array}\right), \quad \mathbf{B}(x)=\left(\begin{array}{c}
B_{1}(x) \\
B_{2}(x) \\
B_{3}(x)
\end{array}\right), \quad x=\left(\begin{array}{l}
x^{0} \\
x^{1} \\
x^{2} \\
x^{3}
\end{array}\right)
$$

in the following. The column vector $x$ denotes Cartesian space-time coordinates $x=\left(x^{0}=c t, x^{1}, x^{2}, x^{3}\right)^{\mathrm{T}}=\left(x^{0}, \mathbf{x}^{\mathrm{T}}\right)^{\mathrm{T}}=\left(x_{0},-x_{1},-x_{2},-x_{3}\right)^{\mathrm{T}}$ in an orthonormal standard coordinate system in Minkowski space. Throughout the paper, we will choose a system of units where the speed of light is $c=1$. 